\title{
SELECTIVE WATER TRANSPORT ACROSS UNIFORM SUB-NANOMETER PORES IN MICROFABRICATED MEMBRANES
}

\author{
T. Humplik ${ }^{1}$, R. Raj ${ }^{1,2}$, S. C. Maroo ${ }^{1,3}$, T. Laoui ${ }^{4}$, and E.N. Wang ${ }^{1 *}$ \\ ${ }^{1}$ Massachusetts Institute of Technology, Cambridge, MA, USA \\ ${ }^{2}$ Currently with: Indian Institute of Technology Patna, Bihar, India \\ ${ }^{3}$ Currently with: Syracuse University, Syracuse, NY, USA \\ ${ }^{4}$ King Fahd University of Petroleum and Minerals, Dhahran, Saudi Arabia
}

\begin{abstract}
We demonstrate selective water transport through uniform sub-nanometer pores using microfabricated zeolite membranes. Despite advances in micro/nanoscale manipulation, creating welldefined sub-nanometer pores for transport studies is challenging. We fabricated the first model platform to characterize and measure water transport limited to $\approx 5.5 \AA$ pores over $>20 \mathrm{~mm}^{2}$ areas. Furthermore, with these membranes, we elucidated the effect of surface chemistry and pore confinement on water permeability. Using a custom-built flow cell, we showed osmotically-driven water transport where a more hydrophobic interface allows for an $\approx 10 \mathrm{x}$ increase in water flux. These insights will help tailor high performance desalination membranes, and can be extended to gas separation, sensing, and energy storage systems.
\end{abstract}

\section{INTRODUCTION}

Membrane-based water desalination has received significant interest due to limited clean water resources [1]. However, current membranes are limited by diffusive transport through tortuous polymeric active layers [1]. By using oriented sub-nanometer pores, water permeability and salt rejection across membranes promise to be substantially increased [2]. Yet, significant challenges in creating such small pores exist, and improved understanding of water transport in sub-nanometer pores is also necessary.

This paper presents a zeolite-based microfabricated membrane to experimentally quantify the water flux and demonstrate size-selective transport. Synthetic MFI zeolites with uniform three-dimensional structures of $\approx 5.5 \AA$ pores, ideal for water transport $(\approx 3 \AA)$ and rejection of hydrated salt ions $(>7 \AA)$, were used. Furthermore, the pore surface chemistry was modified to create more hydrophobic/hydrophilic interfaces.

\section{EXPERIMENTAL APPROACH AND RESULTS}

We prepared two different MFI-type zeolites using hydrothermal synthesis [3]: a hydrophobic (H-MFI) and a more hydrophilic form infused with extra framework sodium cations (Na-MFI). We oriented the zeolites on a commercial anodized aluminum oxide (AAO) membrane $(\mathrm{d}=150 \mathrm{~nm}$, Synkera) using manual direct assembly [4] and evaporated $40 \mathrm{~nm}$ of gold to mask the zeolites during fabrication (Figure 1a). To limit the transport to the sub-nanometer zeolite pores, we utilized the conformality of atomic layer deposition with hafnia to fill the exposed AAO not covered by zeolites (Figure 1b). To expose the zeolites for transport, both reactive ion etching (Figure 1c) and wet etching (Figure 1d) were used. This technique can create membranes $>20 \mathrm{~mm}^{2}$, which allows for the measurement of water transport across $>10^{13}$ sub-nanometer pores (Figure 2).

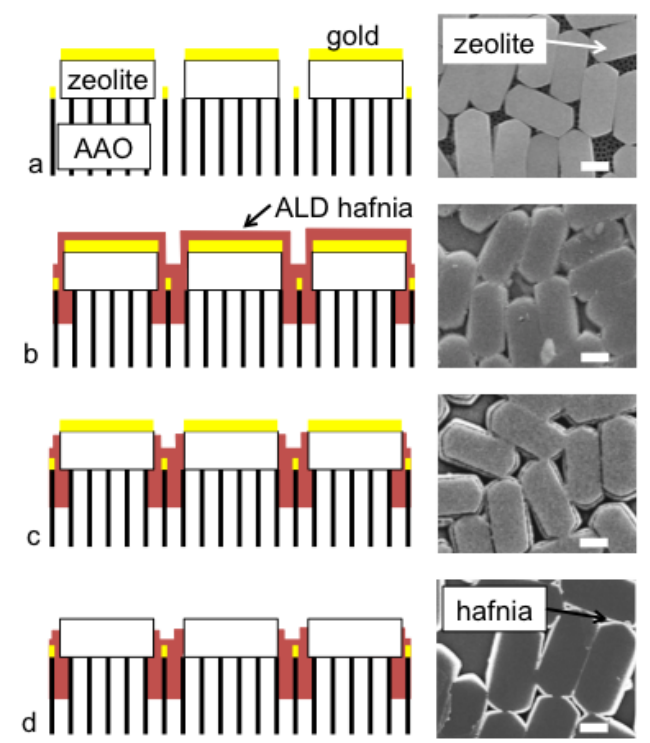

Figure 1: Fabrication schematic (shown with H-MFI zeolites) with corresponding SEM images. (a) Following the zeolite orientation onto the AAO, $40 \mathrm{~nm}$ of gold was thermally evaporated (Sharon Vacuum) onto the membrane to mask the zeolites during processing. (b) The remaining exposed $A A O$ pore structure was filled by depositing hafnia (red in schematic) via atomic layer deposition (Savannah 100, Cambridge NanoTech). (c) Any hafnia that covered the zeolite crystals was dry etched using a $\mathrm{CF}_{4} / \mathrm{O}_{2}$ chemistry (Cirrus 150, Nexx Systems). (d) The gold was wet etched (Gold Etchant, Sigma Aldrich) to expose the underlying subnanometer pore structure and remove any residual hafnia covering the crystals. The scale bar for all images is $1 \mu \mathrm{m}$.
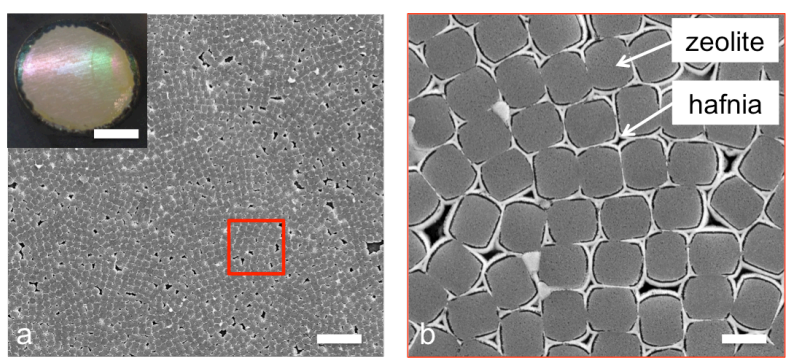

Figure 2: Images of fabricated Na-MFI membranes. (a) Low magnification image demonstrating zeolite orientation over a large area (scale bar is $10 \mu \mathrm{m}$ ). Inset: Image of fabricated membrane on AAO (scale bar is $5 \mathrm{~mm}$ ) (b) Higher magnification image of red boxed region in (a) highlighting both the zeolite (darker cubes) and surrounding ALD hafnia (white regions) (scale bar is $1 \mu \mathrm{m}$ ). 

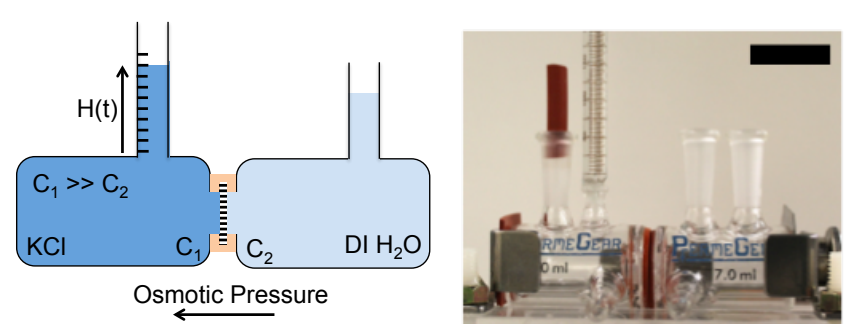

Figure 3: (a) Schematic and (b) image of flow cell used to study water transport across microfabricated zeolite membranes (scale bar is $2 \mathrm{~cm})$. Various molar concentrations $(0.5 M-4 M)$ of potassium chloride was used to generate osmotic pressures of $\approx 2.5 \mathrm{MPa}$ to $\approx 20 \mathrm{MPa}$ to drive the water flux across the membranes. The change in volume (corresponding to a change in height in the graduated cylinder) was monitored as a function of time to quantify the water flux.

We used a custom-built flow cell to investigate water transport using pure water and $\mathrm{KCl}$ (Figure 3). Since the subnanometer zeolite pore structure can exclude the transport of hydrated salt ions [2,5], a selective water flow was generated by a difference in salt concentration (i.e., osmotic pressure).

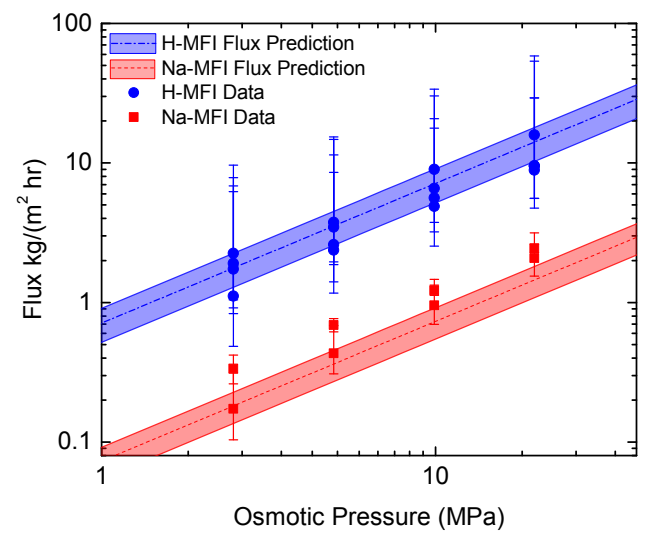

Figure 4: Comparison between experimental data (symbols) and flux prediction (shaded regions) made using transient uptake and desorption analysis (for more details on the model, see [6]). The error bars are associated with the uncertainty in the flux measurements and variation in membrane active area. The linear increase in the flux as a function of the osmotic pressure indicates that water is selectively transporting through the zeolite pores while salt ions are being rejected. The hydrophobic H-MFI zeolites (blue symbols) exhibit $\approx 10 x$ higher water flux than the more hydrophilic Na-MFI zeolites. The decrease in flux associated with the more hydrophilic zeolites is associated with the stronger attraction to the pore interface, which effectively decreases the water diffusivity compared to the hydrophobic interface of the $\mathrm{H}$ MFI zeolites. This insight leads to the possibility of further increasing the flux by up to an order of magnitude by utilizing zeolites that are more hydrophobic than H-MFI.

Figure 4 shows the measured water flux through the membranes compared to our developed model which accounts for the solution-diffusion based transport through the pores [6]. The linear trend of the flux as a function of the osmotic pressure indicates osmotically-driven flow (i.e., the zeolite pores selectively transported water). Furthermore, the flux through the more hydrophobic H-MFI zeolites was $\approx 10 \mathrm{x}$ higher than that of the NaMFI zeolites, contrary to previous zeolite studies [7], and is attributed to the decreased water attraction to the pore surface.
Finally, our good agreement between the experiments and model indicate that the water transport is diffusion-limited, and can be increased further (possibly by an order of magnitude) by enhancing the hydrophobicity of the zeolites.

\section{CONCLUSIONS}

We demonstrated the first device that limits transport to welldefined and oriented sub-nanometer pores over areas $>20 \mathrm{~mm}^{2}$ and showed that pores with a more hydrophobic internal surface facilitate faster water transport. The experimental results from this study can be utilized to provide detailed physical insights into the transport mechanisms, which can guide the design of high permeability membranes in various water-based separation applications.

\section{ACKNOWLEDGEMENTS}

We thank Prof. Michael Tsapatsis (University of Minnesota) and Prof. Rohit Karnik (MIT) for helpful discussions and advice. This work was performed in part at the Center for Nanoscale Systems (CNS), a member of the National Nanotechnology Infrastructure Network (NNIN), which is supported by the National Science Foundation under NSF award no. ECS-0335765. CNS is part of Harvard University. This work was funded by the King Fahd University of Petroleum and Minerals in Dhahran, Saudi Arabia through the Center for Clean Water and Clean Energy at MIT and KFUPM under Project No. R10-CW-09. R.R. acknowledges fellowship support from Battelle's National Security Global Business.

\section{REFERENCES}

[1] T. Humplik, J. Lee, S. C. O’Hern, B. A. Fellman, M. A. Baig, S. F. Hassan, M. A. Atieh, F. Rahman, T. Laoui, R. Karnik, and E. N. Wang, "Nanostructured materials for water desalination," Nanotechnology, vol. 22, no. 29, p. 292001, Jun. 2011.

[2] Y. Liu and X. Chen, "High permeability and salt rejection reverse osmosis by a zeolite nano-membrane," Phys Chem Chem Phys, vol. 15, no. 18, pp. 6817-6824, 2013.

[3] T. Humplik, R. Raj, S. C. Maroo, T. Laoui, and E. N. Wang, "Framework Water Capacity and Infiltration Pressure of MFI Zeolites," Microporous and Mesoporous Materials, 2014.

[4] J. S. Lee, J. H. Kim, Y. J. Lee, N. C. Jeong, and K. B. Yoon, "Manual assembly of microcrystal monolayers on substrates," Angew Chem Int Edit, vol. 46, no. 17, pp. 3087-3090, 2007.

[5] S. Murad, K. Oder, and J. Lin, "Molecular simulation of osmosis, reverse osmosis, and electro-osmosis in aqueous and methanolic electrolyte solutions," Molecular Physics, vol. 95, no. 3, pp. 401-408, 1998.

[6] T. Humplik, R. Raj, S. C. Maroo, T. Laoui, and E. N. Wang, "Effect of Hydrophilic Defects on Water Transport in MFI Zeolites," Langmuir, (submitted)

[7] L. Li, N. Liu, B. McPherson, and R. Lee, "Enhanced water permeation of reverse osmosis through MFI-type zeolite membranes with high aluminum contents," Ind. Eng. Chem. Res, vol. 46, no. 5, pp. 1584-1589, 2007.

\section{CONTACT}

*E.N. Wang, tel: +1-617-324-3311; enwang@mit.edu 